\title{
Avaliação dos erros ou falhas de preenchimento dos atestados de óbito feitos pelos clínicos e pelos patologistas
}

\author{
Comparison between death certificates filling errors or lack of filling fields done by pathologists and physicians
}

\author{
Cleber de Oliveira Stuque ${ }^{1}$ \\ José Antônio Cordeiro² \\ Patricia Maluf Cury ${ }^{3}$
}

\begin{abstract}
unitermos resumo
Autópsia

Atestado de óbito

Introducão: O preenchimento correto dos atestados de óbito (AO) é muito importante, porém geralmente negligenciado. Objetivo: Comparar os $\mathrm{AO}$ preenchidos pelos patologistas e pelos clínicos em relação à quantidade de erros de preenchimento e áreas não-preenchidas. Método: Foram comparados os atestados fornecidos pelo serviço de cardiologia do Hospital de Base (HB) e os das autópsias do Serviço de Verificação de Óbitos (SVO), de causas cardíacas, no ano de 2000, num total de $282 \mathrm{AO}$. Foi considerado erro ou falha de preenchimento quando os dados não correspondiam à verdade (p. ex.: item só para mulheres preenchido em paciente masculino) ou quando havia falta de preenchimento de algum item. Resultados: Houve diferença em relação às médias das idades entre 0 grupo HB (67,8 anos) e o SVO (61,9 anos; $p<0,001)$. No grupo HB não houve nenhum AO com todos os campos preenchidos, enquanto no grupo SVO 11,92\% estavam completamente preenchidos. Entretanto $50 \%$ dos $\mathrm{AO}$ do grupo $\mathrm{HB}$ tinham apenas um erro ou falta de preenchimento e somente 7,6\% tinham cinco erros ou mais. Já no grupo SVO, 21,85\% dos AO possuíam cinco erros ou mais. Nos campos assistência médica recebida, exame complementar realizado e cirurgia, o grupo SVO obteve mais erros que o grupo $\mathrm{HB}(p<0,001)$. $\mathrm{O}$ item tempo de evolução da doença não foi preenchido em nenhum dos AO do grupo HB e em apenas $31,7 \%$ dos casos do $\mathrm{SVO}(p<0,001)$. Conclusão: Os patologistas apresentaram mais falhas no preenchimento do $\mathrm{AO}$ que os clínicos.

Introduction: The correct filling of Death Certificate $(D C)$ is very important, however is usually neglected.

Objectives: To compare DC filled by the pathologists and by the physicians, in relation to the number of mistakes and also the number of fields not filled. Methods: We compared all DC filled by physicians from the

Hospital de Base (HB) and DC filled by pathologists from the Pathology Department (PD), after autopsy procedure, during the year of 2000 ( $\mathrm{n}=282)$. A filling error was defined as an item not filled correct (ex.: only for women and filled in a male patient) or not filled at all. Results: The mean age were different comparing $H B$ group (67,8 years) and PD group $(61,9$ years; $p<0.01)$. In HB group none of the $D C$ was completely filled, while in PD 11.92\% were filled correctly (with all fields filled). However, $50 \%$ of the HB filled DC had only one

mistake and $7.6 \%$ had five or more errors, while in PD group $21.85 \%$ had five or more errors. In the items medical assistance, complementary exams and surgery of the DC, PD group had more non-filled fields than $H B(p<0.001)$. In relation to the item interval time of disease's evolution, no DC from HB group filled it, and just $31.7 \%$ of the pathologist's DC had it filled. Other kind of mistakes were observed and described. Conclusion: Pathologists had more fields not filled than the physicians did.
Autopsy

Death certificate

Errors

1. Aluno do quinto ano de Medicina da Faculdade de Medicina de São José do Rio Preto (Famerp), com bolsa de iniciaçãa científica pela Fundação de Amparo à Pesquisa do Estado de São Paulo (Fapesp).

2. Bioestatístico; professor doutor do Departamento de Epidemiologia e Saúde Coletiva da Famerp. 3. Médica patologista; professora doutora do Departamento de Patologia e Medicina Legal da Famerp.

Departamento de Patologia e Medicina Legal da Faculdade de Medicina de S. I. do Rio Preto, São Paulo. 


\section{Introducão}

As estatísticas de mortalidade constituem ferramenta de grande valor em administração sanitária, demográfica e epidemiológica. Entretanto sabe-se que nem sempre correspondem aos verdadeiros fatos ocorridos, principalmente com relação à causa da morte $(3,4)$. Entre várias justificativas, merece destaque a falta de atenção, por parte dos currículos das escolas médicas, ao ensino do correto preenchimento do atestado de óbito $(\mathrm{AO})$ e à sua importância como ferramenta de dados para a saúde pública. É comum médicos se depararem, pela primeira vez, com um atestado de óbito no momento em que se vêem na contingência real de preenchê-lo. Conseqüentemente ocorrem, não raras vezes, falhas no preenchimento. Laurenti e Fonseca (2) colheram várias informações que permitiram conhecer a verdadeira causa básica da morte da certos pacientes e compararam com o respectivo AO. Foram verificadas falhas no preenchimento de vários itens, entre eles intervalo de tempo entre o início da doença e a morte, exames complementares, verdadeira causa básica, entre outros.

Além da importância da realização da autópsia para melhorar o diagnóstico da causa da morte, fato ainda muito discutido na literatura $(1,6,7)$, aparentemente, na nossa opinião, os $\mathrm{AO}$ são mais bem preenchidos pelos patologistas do que pelos clínicos, tanto pela falta de experiência no assunto dos últimos como também pelo maior número de informação que supostamente o patologista teria após a realização da autópsia.

$\mathrm{O}$ intuito deste trabalho foi tentar confirmar esta suspeita, comparando-se os atestados de óbito preenchidos pelos patologistas e pelos clínicos, não em relação ao tipo de diagnóstico, mas sobre a forma de preencher $\mathrm{o}$ atestado e a quantidade de erros e falhas de preenchimento.

\section{Materiais e métodos}

Para restringir a área de atuação, foram comparados os atestados fornecidos pelo Serviço de Cardiologia do Hospital de Base de São José do Rio Preto (HB) e as autópsias do Serviço de Verificação de Óbitos (SVO) de pacientes com idade superior a 12 anos, oriundos do mesmo hospital no ano de 2000, com óbito por causas cardíacas. Todos os pacientes possuíam prontuários disponíveis para a realização da necropsia, contendo todos os dados necessários para o preenchimento do atestado de óbito no momento da mesma. A análise dos dados foi baseada nas regras de preenchimento do atestado de óbito fornecidas pelo Centro da OMS para classificação de doenças em português (5). Foi analisado o correto preenchimento de diversos campos e considerados erros ou falhas de preenchimento: campos preenchidos incompletamente (p. ex. prenchimento do item $a$, mas não do $b$ ); não-preenchimento (deixados em branco); preenchimento inadequado (na verdade deveriam ficar em branco: p. ex., campo destinado a mulheres preenchidos por dados de pacientes do sexo masculino) e campos onde os dados eram ilegíveis. O AO foi considerado completo quando todos os campos foram preenchidos corretamente.

Seis patologistas realizaram as autópsias no serviço e preencheram os $\mathrm{AO}$, todos com pelo menos dez anos de experiência e formação em patologia em diferentes centros. Quanto aos clínicos, a variação é muito grande, havendo desde residentes até cardiologistas com mais experiência, dificultando a melhor caracterização do grupo. $O$ método de avaliação estatística usado foi o de estimação e comparação de frações defeituosas entre os dois grupos de atestados de óbito, tendo sido procedida por comparação de proporções, pelo método exato, quando era o caso. A comparação do número de campos preenchidos foi feita por meio de teste não-paramétrico de MannWhitney, e o número de erros ou falhas de preenchimento foi verificado por teste $t$ de Student.

\section{Resultados}

Foram avaliados 282 atestados de óbito, 141 do SVO e 141 do HB. A média de idades no grupo HB (atestados preenchidos pelos médicos do HB) foi de 67,8 anos $(s=16,29$; mediana $=63,9$, mínima de 20 e máxima de 96 anos) e no grupo SVO (atestados preenchidos pelos patologistas após a necropsia), de $61,9(s=15,42$; mediana $=59,4$, mínima de 17 e máxima de 94 anos $)(p=0,001)$. Num total de 163 casos masculinos, 96 (58,8\%) vão para - SVO para ser realizada a autópsia contra $41 \%$ do sexo feminino (55 casos num total de 132 pacientes), ou seja, nos pacientes cardíacos que vêm para o hospital, há evidência de que homens são mais submetidos a autópsia que as mulheres $(p=0,001)$.

No grupo HB não houve $A O$ sem erros ou com falha no preenchimento, isto é, com todos os campos preenchidos corretamente, enquanto no grupo SVO, 11,92\% dos atestados estavam completos e sem erros ou sem falha no preenchimento. Entretanto $50 \%$ dos AO do grupo HB tinham apenas um erro ou falha no preenchimento. Em todos estes casos, o erro foi a falta de preenchi- 
mento do campo tempo de evolução da doença - item 49 do $\mathrm{AO}$. Enquanto no grupo $\mathrm{HB} 7,7 \%$ dos $\mathrm{AO}$ tinham, no mínimo, cinco erros ou falha no preenchimento, no grupo SVO esta percentagem era de $21,85 \%$. A Tabela relaciona o número de erros ou falha no preenchimento com a porcentagem de aparecimento dos mesmos, tanto no HB quanto no SVO.

A Figura ressalta os campos relacionados com assistência médica recebida (45), exame complementar realizado (46) e cirurgia (47). O grupo SVO obteve mais erros que o grupo HB: $64,90 \%, 56,95 \%, 47,68 \%$ vs. $25,7 \%$, $8,33 \%, 10,42 \%$, respectivamente (todos os itens com $p<0,001)$. Nos demais campos, não houve diferença significante entre os dois grupos. Os campos 43 (óbitos de mulheres durante gravidez, parto ou aborto) e 44 (óbitos de mulheres durante o puerpério) foram quase sempre preenchidos corretamente nos dois grupos e ficou demonstrado que tanto o grupo SVO como o HB obtiveram mais acertos que erros nestes campos. Entretanto, em dois casos de pacientes masculinos do grupo HB, estes campos foram preenchidos.

Com relação ao item tempo, do campo 49 , nenhum $\mathrm{AO}$ do grupo $\mathrm{HB}$ o tinha preenchido, enquanto apenas $31,7 \%$ do grupo SVO o tinham $(p<0,001)$ (cabe relembrar

\section{Número de erros por atestado}

\section{Tabela de óbito}

\begin{tabular}{lcccccccc}
\hline $\begin{array}{l}\text { Número } \\
\text { de erros }\end{array}$ & 0 & 1 & 2 & 3 & 4 & 5 & 6 & 7 \\
HB (\%) & - & 50 & 19,4 & 16,67 & 6,25 & 4,9 & 1,4 & 1,4 \\
SVO (\%) & 11,92 & 18,5 & 6,62 & 15,89 & 25,2 & 11 & 6,6 & 4 \\
\hline
\end{tabular}

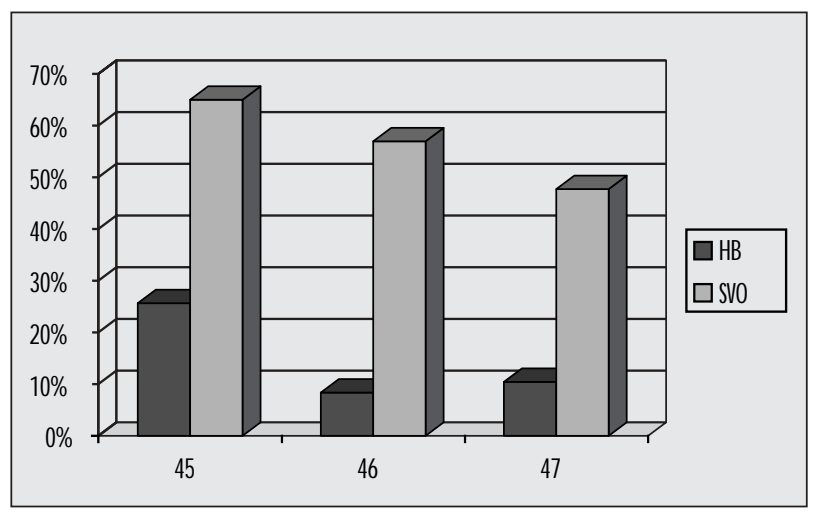

Figura - Erros nos itens 45, 46, 47 versus porcentagem de aparecimentos nos dois grupos que estas informações estavam disponíveis nos prontuários dos pacientes no momento da necropsia).

Em três casos do grupo HB o AO estava ilegível por má caligrafia no momento do preenchimento do documento.

\section{Discussão}

Os resultados deste trabalho foram de certa maneira diferentes do esperado. Em primeiro lugar, a diferença entre a média de idade dos dois grupos, em que os pacientes do grupo SVO submetidos a necropsia eram significantemente mais novos do que os do grupo $\mathrm{HB}$, sugere que as pessoas que morrem mais tardiamente, por terem na maioria das vezes doenças crônicas e acompanhamento médico por maior tempo, provavelmente já têm a causa da morte mais definida. Já pessoas que morrem mais cedo têm menor acompanhamento médico e, na maioria das vezes, é necessária a autópsia para desvendar a causa da morte, explicando o dado acima.

Em relação ao maior número de pacientes do sexo masculino submetidos a autópsia, não encontramos nenhuma explicação plausível. Talvez, se o número de casos fosse maior, este dado pudesse não ter significância estatística. Por último, o mais importante e o objetivo maior do trabalho foi a identificação de uma grande falha de preenchimento dos atestados feitos pelos patologistas. $\mathrm{Na}$ verdade, não foi considerada a qualidade de preenchimento, pois os pacientes não eram os mesmos e apenas a quantidade de erros foi observada. Os patologistas erram principalmente na falta de preenchimento de itens (principalmente do 43 ao 48), o que poderia ser explicado pela atenção apenas no preenchimento da causa da morte (campo 49). Os itens anteriores são negligenciados muitas vezes por se esperar que a secretária os preencha, o que geralmente não ocorre com os clínicos, já que eles acabam tendo que preencher todo o $\mathrm{AO}$ sem ajuda de outras pessoas. O campo tempo aproximado entre o início da doença e a morte não foi preenchido por nenhum dos clínicos e em apenas $11 \%$ dos $\mathrm{AO}$ dos patologistas, o que demonstra um desconhecimento quase total, por parte dos médicos, de sua importância. Erros que consideramos grosseiros foram observados no preenchimento de alguns itens, como dados sobre óbitos de mulheres (itens $43 \mathrm{e}$ 44) em pacientes do sexo masculino e letras ilegíveis, que ocorreram mais nos $\mathrm{AO}$ fornecidos pelos clínicos. Quanto ao preenchimento das causas de morte, embora não fosse o objetivo de nosso trabalho, foi observado que os clínicos não colocam como causa básica a aterosclerose na maioria dos casos de óbito cardíaco por infarto agudo do miocárdio 
(dado não-mostrado), deixando como causa básica apenas o infarto. Não foi realizada a análise comparativa dos diagnósticos de causa de óbito, pois não só os pacientes eram distintos, como tanto o corpo clínico quanto o de patologistas eram muito heterogêneos, o que não permitiria tal comparação. Entretanto um outro trabalho de nosso grupo fez esta análise avaliando o mesmo paciente, a qual está sendo submetida à publicação. Tudo isto nos mostra que ainda existe muita dificuldade no preen- chimento do AO por parte dos médicos e, inclusive, dos patologistas. Uma maior atenção a este documento deve ser dada durante a formação médica, pois suas informações são de suma importância para a saúde pública.

\section{Agradecimentos}

Ao prof. dr. Moacir Godoy e à Fapesp, pelo apoio e pela bolsa de iniciação científica.

\section{Referências}

1. Carvalho, F.M. et al. C linical diagnosis versus autopsy. Bull Pan Am Health Organ, 25:41-6, 1991.

2. Fonseca, L.A.M. \& Laurenti, R.A qualidade da certificação médica da causa de morte em São Paulo, Brasil. Saúde Púb., 8: 21-9, 1974.

3. G uite, H .F. \& Burney, P.G .J.A ccuracy of recording of deaths from asthma in the UK: the false negative rate. Thorax, 51:9248, 1996 .

4.Kircher,T;,N elson,J.\& Burdo,H.The auto psy as a measure of accuracy of death certificate. N . Eng.l J. Med., 313: 1263-9,1985.
5. Laurenti, R. \& Jorge, M.H.P.M. 0 atestado de óbito. Centro da O MS para a Classificação de Doenças em Português. 3. ed., 1996.

6.Valdez-M artinez, E.;A Arroyo-Lunagomez, E. \& Landero-Lopez, L. Concordancia entre el diagnóstico clínico y el patológico por necropsias. Salud Pública de M éxico, 40:32-7,1998.

7. Zarbo, R.J.; Baker, P.B. \& Howanitz, P.J.The autopsy as a performance measurement tool: diagnostic discrepancies and unresolved clinical questions. Arch. Pathol. Lab. Med., 123: 191-8, 1999. 